\title{
Reaching Consensus in Multiagent Decision Making
}

\author{
Antonio Maturo \\ University of Chieti - Pescara \\ antmat@libero.it \\ Aldo G. S. Ventre \\ University of Napoli II \\ aldoventre@yahoo.it
}

\begin{abstract}
In a dynamic context, group decisions result as a mixture of agreement and compromise. We will put in evidence that an average of judgements of the components, $i$. e. the decision makers, of a committee can be built only if a majority of the members not too different opinions. We assume that an external chairman, with a complete information about the state of all the components of group, urges or invites decision makers to reach a sufficient consensus. In this paper, the judgements of experts are represented by points of a metric space, and the consensus is obtained by a dynamical construction of a maximal winning coalition contained in a ball with a fixed and suitably small diameter.
\end{abstract}

\section{Keywords: Multiperson Decision Making, Cooperative Games, Consensus, Metric Spaces}

\section{Consensus in Multiagent Decision Making}

Not always the opinions are clearly settled in the individual. Sometimes the opinions are uncertain, and can be influenced and modified by other individuals. So, as in a usual discussion among the members of a group, especially the experts or decision makers of a committee, it happens that the participants modify their points of view, as a consequence of the entering of pieces of information, or the opinions expressed by others. Often someone tries to change the ideas of the individuals that have different positions in the group, and the reactions are not uniform: some individuals defend and confirm their own position, others admit the onset of doubts and uncertainties, and others modify, totally or in part, their previous opinions. Moreover someone does not want to be emarginated from the group and asks for modify his own opinion in the direction of the dominant opinion in the group. [Carlsson et al., 1992; Corrao, 2000].

This is related with any description or definition of the behavior of the components in a group whose aim is to reach consensus.

Let us illustrate some reasonable issues that the concept of consensus should meet (see, e. g., Carlsson et al.,1992, Eklund et al., 2007).

Seeking consensus, especially if utility issues are involved, is the result of a trade-off between agreement and compromise in the behavior of a single decision maker. This introduces a dynamic procedure toward consensus. More precisely, consensus is reached by a group of persons that do their best in order to obtain a prefixed goal or a good result in some direction. Furthermore, usually group decisions are made by a majority of the members in the committee: the more numerous is the majority, the higher is the degree of consensus reached by the group. Indeed, unanimous group decisions are rare in real life.

Moreover seeking consensus requires a perfect information about the behavior of any other member at disposal of each member in the group.

It is assumed that a chairman or supervisor, external to group, coordinates the group decision process. In the real life meetings the chairman plays a role such as: he summarizes the discussion, thus defining the current status, repeating characteristic opinions, and implicitly pointing at contradicting views in diplomatic way, recalling the need to maintain the group. 
Those who feel being opponents and fear being left out from some majority react by asking to clarify or modify their opinions so as to weaken the tension between their own views and what it is concluded to be the current state of consensus.

Our present aim is to exhibit a game theoretic point of view related with the dynamics of a consensus reaching process. We look at consensus as a construction of a winning coalition in a cooperative game where:

i) the decision makers are the players;

ii) utility transfer is allowed;

iii) only suitable coalitions are admissible.

\section{A Model of Multiperson Decision Making}

Now we describe a model of collective decision making and the related consensus achievement procedure (see also, e. g. Carlsson et al., 1992, and Eklund et al., 2007).

Let $\mathrm{D}=\left\{\mathrm{d}_{1}, \mathrm{~d}_{2}, \ldots, \mathrm{d}_{\mathrm{n}}\right\}$ be the set of the decision makers of a committee, $\mathrm{A}=\left\{\mathrm{a}_{1}, \mathrm{a}_{2}, \ldots, \mathrm{a}_{\mathrm{m}}\right\}$ the set of alternatives, and $\mathrm{C}=\left\{\mathrm{c}_{1}, \mathrm{c}_{2}, \ldots, \mathrm{c}_{\mathrm{p}}\right\}$ the set of the accepted criteria. Let any decision maker $\mathrm{d}_{\mathrm{r}} \in \mathrm{D}$ be able to assess the relevance of each criterion $\mathrm{c}_{\mathrm{j}}$ with a non negative real number $\mathrm{w}_{\mathrm{j}}{ }^{(\mathrm{r})}$, with the normality conditions: for every $r \in\{1,2, \ldots, n\}, \Sigma_{j} \mathrm{w}_{\mathrm{j}}^{(\mathrm{r})}=1$.

Moreover, for every alternative $a_{i}$ and criterion $c_{j}$, let $s_{i j}{ }^{(r)}$ be the score assessed by the expert $d_{r}$. We assume the $\mathrm{s}_{\mathrm{ij}}{ }^{(\mathrm{r})}$ are real numbers belonging to the interval $[0,1]$.

In order to give a geometrical representation of the preferences of the set of decision makers in the geometric space of the alternatives, to every expert $d_{r}$ is associated the point $P_{r}$ of $R^{m}$ with coordinates:

$$
\mathrm{u}_{\mathrm{i}}^{(\mathrm{r})}=\Sigma_{\mathrm{j}} \mathrm{w}_{\mathrm{j}}^{(\mathrm{r})} \mathrm{s}_{\mathrm{ij}}^{(\mathrm{r})}, \mathrm{r} \in\{1,2, \ldots, \mathrm{m}\}
$$

Because of the conditions $\mathrm{w}_{\mathrm{j}}{ }^{(\mathrm{r})} \in[0,1], \mathrm{s}_{\mathrm{ij}}{ }^{(\mathrm{r})} \in[0,1], \Sigma_{\mathrm{j}} \mathrm{w}_{\mathrm{j}}^{(\mathrm{r})}=1$, every point $\mathrm{P}_{\mathrm{r}}$ belongs to the unit cube of $\mathrm{R}^{\mathrm{m}}$.

Dynamics of consensus enhancing process is managed by the chairman by introducing in $\mathrm{R}^{\mathrm{m}} \mathrm{a}$ metric, e. g. the Euclidean metric, that acts between couples of points of $\mathrm{R}^{\mathrm{m}}$ associated to decision makers. For instance, (see Carlsson et al., 1992, and Eklund et al., 2007), we can assume that the distance between the decision makers $d_{r}$ and $d_{s}$ is given by the formula:

$$
\mathrm{d}_{\mathrm{rs}}=\sqrt{\frac{1}{\mathrm{~m}} \sum_{\mathrm{i}=1}^{\mathrm{m}}\left(\mathrm{u}_{\mathrm{i}}^{(\mathrm{r})}-\mathrm{u}_{\mathrm{i}}^{(\mathrm{s})}\right)^{2}} .
$$

The supervisor observes, at any step of the decision making process, the position of each member in the committee and informs the more peripheral expert about the opportunity to revise his judgement.

If we set $\mathrm{d}^{*}=\max \left\{\mathrm{d}_{\mathrm{rs}} \mid \mathrm{r}, \mathrm{s} \in\{1,2, \ldots, \mathrm{n}\}\right\}$, then a measure of the degree of consensus $\gamma$ can be defined as the complement to one of the maximum distance between two positions of the experts:

$$
\gamma=1-\mathrm{d}^{*}=1-\max \left\{\mathrm{d}_{\mathrm{rs}} \mid \mathrm{r}, \mathrm{s} \in\{1,2, \ldots, \mathrm{n}\}\right\} .
$$

The chairman can consider a level $\delta \in(0,1)$ and assume there is consensus if $\mathrm{d}^{*}<\delta$, that is $\gamma>1$ $\delta$. Alternatively, the supervisor can judge that consensus al level $\delta$ means that decision makers belong to a ball of diameter $\delta$ of the metric space $\mathrm{R}^{\mathrm{m}}$. This point of view will be considered in the next section. 


\section{Reaching Consensus in a Game Theoretic Point of View}

We describe reaching consensus as a dynamical procedure of movement of points in a metric space. We assume that, at every step, the members of the committee $\mathrm{D}$ that must reach consensus are represented as points of a given metric space $(\mathrm{S}, \mathrm{d})$ and that such points can change their positions in the successive step. At step $\mathrm{h}$ the consensus is obtained if a "sufficient number" of members of the committee are contained in a "small ball" of (S, d). Moreover, we relate consensus with the construction of a winning coalition in a cooperative game where players are decision makers and utility transfer is allowed.

Let $\mathrm{n}, \mathrm{n} \geq 3$, be the number of decision makers. For every integer $\mathrm{k}$, with $\mathrm{n} / 2<\mathrm{k} \leq \mathrm{n}$, a set of components of the committee with at least $\mathrm{k}$ elements is said to be a majority at level $\mathrm{k}$.

Let now $\delta$ be a positive real number, called level of proximity. We say that q members in the committee agree at level $\delta$ if they belong to a ball of diameter $\delta$ of the metric space (S, d).

We introduce the following definitions:

- a subset $\mathrm{H}$ of $\mathrm{D}$ is said to be an admissible coalition, at level $\delta$ and at time $\mathrm{h}$, if at step $\mathrm{h}$ the elements of $\mathrm{H}$ are points of $(\mathrm{S}, \mathrm{d})$ contained in at least a ball of diameter $\delta$ of the space;

- an admissible coalition $\mathrm{H}$ is said to be a winning coalition at level $\mathrm{k}$ if $\mathrm{H}$ is a majority at level k.

The important concept of admissible coalition was considered also by many authors, in particular in Luce, Raiffa (1957). Admissibility was related with constraints that were in nature ethical, social, etc. Our present point of view is very different because we introduce a geometrical interpretation of admissibility concept in order to describe "a sufficient proximity" of the components of a coalition.

We remark that, for every $\delta>0$, the set of admissible coalitions at level $\delta$ is not empty because every singleton is contained in at least a ball of diameter $\delta$.

From now on, we assume that the committee $\mathrm{D}$ agree on the level of majority $\mathrm{k}$ and on the level of proximity $\delta$ and then in the sequel we don't recall such levels.

At step $\mathrm{h}$, let $\mathrm{K}$ be the set of the admissible coalitions. A not winning coalition $\mathrm{H}$, whose elements are in number of $|\mathrm{H}|$, belonging to $\mathrm{K}$, is said to be:

i. losing, if the coalition $\mathrm{H}^{\mathrm{c}}=\mathrm{D}-\mathrm{H} \in \mathrm{K}$ contains a winning coalition;

ii. quasi-losing, if $\left|\mathrm{H}^{\mathrm{c}}\right| \geq \mathrm{k}$, but $\mathrm{H}^{\mathrm{c}}$ does not contain any winning coalition;

iii. blocking, if $|\mathrm{H}|<\mathrm{k}$ and $\left|\mathrm{H}^{\mathrm{c}}\right|<\mathrm{k}$.

The concepts of winning and losing coalitions were studied also in Shapley (1962), where simple games are considered. It is worth observing that, while in simple games all coalitions are considered admissible, in this paper, we assume as admissible only the coalitions satisfying suitable geometric constraints. In particular, in simple games the whole group of players is a winning coalition, on the contrary in our framework a coalition with at least $\mathrm{k}$ members is winning if and only if it is included in a ball of diameter $\delta$. Moreover, we consider the new concept of quasi-losing coalition.

A fundamental difference between the classical, and also fuzzy, game theory and our framework is that in the first one minimal winning coalitions are considered (Shapley, (1962), Luce, Raiffa, (1957), Mares, (2001)), while in the second one finding the consensus means to look for maximal winning coalitions.

In the sequel, every maximal winning coalition of $\mathrm{K}$ is said to be $a$ solution of the consensus reaching problem. We introduce the following further

At step $\mathrm{h}$ one of the following cases occurs:

(1) $\mathrm{D} \in \mathrm{K}$;

(2) $\mathrm{D} \notin \mathrm{K}$, but the consensus problem has a unique solution;

(3) $\mathrm{D} \notin \mathrm{K}$ and the consensus problem has at least two solutions; 
(4) $\mathrm{D} \notin \mathrm{K}$ and the consensus problem has no solutions.

Reaching a sufficient consensus can be interpreted as to have a final solution $\mathrm{D}^{*}$ that the chairman thinks cannot be enlarged in the successive step. Then, in the case (1), a sufficient consensus is reached, with the maximum number of elements.

In the case (2), if $\mathrm{H}$ is the unique maximal solution, the chairman either assumes as final solution $\mathrm{D}^{*}=\mathrm{H}$ or tries to enlarge the set $\mathrm{D}^{*}$, by persuading some members of $\mathrm{H}^{\mathrm{c}}=\mathrm{D}-\mathrm{H}$ to change their evaluations, that is to move in the metric space $(\mathrm{S}, \mathrm{d})$. In the second alternative can happen that the players not belonging to the ball of diameter $\delta$ containing $\mathrm{H}$ don't move in order to fall in this ball, but near to this ball, in such a way to form, with some elements of $\mathrm{H}$, a maximal winning coalition $H^{\prime}$ not containing $H$. The aim is to activate a strategy to exclude particular elements of $\mathrm{H}$ from the final decision.

In the case (3), the chairman either applies some criteria to choose as final solution $\mathrm{D}^{*}$ one among the solutions at step $h$ or tries to enlarge consensus by persuading the members of $D$ not belonging to all the solutions to change their evaluations.

Finally, in the case (4), the chairman either tries to persuade the members of D to get closer or decides that it is not possible to have a solution of the to consensus problem.

We call a move at step h every change of evaluation of the members of $\mathrm{D}$ not belonging to all the solutions. Let $\mathrm{D}_{\mathrm{h}}=\left\{\mathrm{P}_{1}, \mathrm{P}_{2}, \ldots, \mathrm{P}_{\mathrm{n}}\right\}$ the set of points of $(\mathrm{S}, \mathrm{d})$ that represent the players at step $\mathrm{h}$. The set of the $n(n-1) / 2$ real numbers $U\left(D_{h}\right)=\left\{d\left(P_{i}, P_{j}\right), i<j\right\}$ is the vector distance at step $h$.

A function $\mathrm{f}:[0,+\infty)^{\mathrm{n}(\mathrm{n}-1) / 2} \rightarrow[0,+\infty)$ is said to be an admissibility criterion if it is increasing with respect to every variable and it is invariant with respect every permutation of the variables.

A move from $\mathrm{D}_{\mathrm{h}}=\left\{\mathrm{P}_{1}, \mathrm{P}_{2}, \ldots, \mathrm{P}_{\mathrm{n}}\right\}$ to $\mathrm{D}_{\mathrm{h}}^{\prime}=\left\{\mathrm{P}_{1}{ }_{1}, \mathrm{P}_{2}^{\prime}, \ldots, \mathrm{P}_{\mathrm{n}}^{\prime}\right\}$ is said to be admissible, with respect to the admissible criterion $\mathrm{f}$ and to threshold $\varepsilon>0$, if $\mathrm{f}\left(\mathrm{U}_{(}\left(\mathrm{D}_{\mathrm{h}}^{\prime}\right)\right)<\mathrm{f}\left(\mathrm{U}_{(}\left(\mathrm{D}_{\mathrm{h}}\right)\right)-\varepsilon$.

Suppose chairman and players agree on an admissibility criterion $\mathrm{f}$ and a threshold $\varepsilon>0$. Then the chairman decides that there are not possible move if the players are not willing for any admissible move.

A very particular case is the one considered in the papers Carlsson et al., 1992, and Eklund et al., 2007, that we call the Bastian procedure.

An alternative to the Bastian procedure is to decide that the maximal winning coalition to enlarge is the one with the maximum number of players. If there are more winning coalitions with these properties, the coalition to be enlarged is the one, if it is unique, that is included in a ball of minimum diameter.

We can also consider fuzzy coalitions as in Mares (2001), and their fuzzy widths.

We remark that a serious obstacle in order to obtain a solution can arise by the formation of blocking coalitions. Then the existence of blocking coalitions may induce some corrections to our procedures, such as an activation of a form of bargaining or an evaluation of the power of these coalitions.

\section{Bibliography}

Carlsson C., Ehrenberg D., Eklund P., Fedrizzi M., Gustafsson P., Lindholm P., Merkurieva G., Riissanen T., Ventre A. G. S. (1992), Consensus in distributed soft environments, European Journal of Operational Research 61, 165-185.

Corrao S., Il focus group, Franco Angeli, Milano, 2000.

Eklund P., Rusinowska A., De Swart H.(2007), Consensus reaching in committees, European Journal of Operational Research 178, 2007, 185-193.

Luce R. D., Raiffa H. (1957), Games and Decisions, John Wiley, New York.

Mares M. (2001), Fuzzy Cooperative Games, Springer Verlag, New York.

Saaty T. L., (1980), The Analytic Hierarchy Process, McGraw-Hill, New York, 1980.

Shapley L. S. (1962), Simple games. An outline of the theory, Behavioral Sciences 7: 59-66. 